\title{
Über die Wirkung von oralen Antidiabetica auf den Blutinsulingehalt der Hunde und der Ratten.*
}

\author{
Von
}

\section{Genji NAGATA}

Aus der pharmakologischen Abteilung des pharmazeutischen Instituts Shionogi, Amagasaki, Japan.

\author{
(Leiter : Dr. T. Minesita)
}

Seitdem Janbon et $\mathrm{al}^{1}{ }^{1}$ im Jahre 1942 die blutzuckersenkende Wirkung des Isopropylthiodiazol-Derivats von Sulfonamid (2254 RP) entdeckt hatten, wurde die Forschung nach antidiabetischen Substanzen fortgesetzt. 1955 berichteten die Forscher in Deutschland über die blutzuckersenkende Wirkung von $\mathrm{N}_{1}$-Sulfanilyl- $\mathrm{N}_{2}$-(n-butyl)-Harnstoff ${ }^{23) 3) \text { () }}$ (Carbutamide oder BZ55) und $\mathrm{N}_{1}$-p-Toluolsulfonyl- $\mathrm{N}_{2}$-(n-butyl)-Harnstoff ${ }^{\text {j) }}$ (Tolbutamide oder D860). Andererseits entdeckten Kobayashi ${ }^{6)}$ et al., Ōhashi ${ }^{7 / s)}$ et al., in Japan im Jahre 1952 die antidiabetische Wirkung von Mesoxalate.

Diese Substanzen werden jetzt zur Behandlung der Zuckerkrankheiten verwendet. Aber die Kenntnis vom Wirkungsmechanismus der Blutzuckersenkung durch die oralen Antidiabetica von Sulfonamid-Derivat geht nicht über die Grenzen der Hypothese hinaus. Sie lassen sich in zwei Hauptklassen einteilen, nämlich 1) Pankreatotrope Theorie, 2) Hepatotrope Theorie. Wir unternahmen die folgenden Experimente mittels der neuen Methode über die Bestimmung des Blutinsulingehaltes, um etwas zur Aufklärung des Wirkungsmechanismus von den oralen Antidiabetica beizutragen.

\section{Methodik}

1) Die Bestimmungsmethode des Insulingehaltes im Blut (festbegründet von Minesita ${ }^{9)}$ et al.). Da die Einzelheiten dieser Methode schon von Minesita et al. berichtet worden sind, stellen wir sie an dieser Stelle nur kurz dar.

a) Die Methode der Extraktion von Insulin aus dem Blutplasma.

Einige Modifikationen wurden von der von Best $^{10)}$ et al. festgestellten Methode gemacht, welche den besten Einsammlungssatz im Falle der Extraktion von Insulin aus dem Pankreas im Vergleich mit anderen Methoden besitzt, um die Ausbeute von Insulin aus dem Blut noch mehr zu verbessern.

$3.0 \mathrm{ccm}$ von Blutplasma oder Serum werden mit $50 \mathrm{ccm}$ von $75 \%$ igem saurem Alkohol gemischt, welcher aus einem Gemisch von $1.5 \mathrm{ccm} \mathrm{HCl}$ pro $100 \mathrm{ccm}$ von $75 \%$ igem Alkohol

\footnotetext{
* Der Kernpunkt dieser Abhandlung wurde am 5. Oktober 1958 in Tottori beim sechsten Kongress der west-japanischen Abteilung der japanischen endokrinologischen Gesellschaft und am 31. Oktober 1958 in Osaka beim 16-ten Kongress der Kinki-Abteilung der japanischen pharmakologischen Gesellschaft veröffentlicht.
} 
besteht. Der mit Plasma gemischte sauere Alkohol wird dann unter $36^{\circ} \mathrm{C} 2$ Stunden lang geschüttelt.

Filtration mit Filtrierpapier.

Das Residuum wird mit dem im Stücken geschnittenen Filtrierpapier durch den vorgenannten saueren Alkohol reextrahiert. Die Verdampfung des Alkohols aus dem eingesammelten Filtrat wird unter $35^{\circ} \mathrm{C}$ im Zustand des Vakuums vorgenommen. Die Fraktion von Insulin wird dann durch die Hinzufügung von $50 \sim 100 \mathrm{ccm}$ der gesättigten Kochsalzlösung zu dem vom Alkohol befreiten trüben Saft nach 2 Stunden ausgesalzen. Die Fällung der ausgesalzten Fraktion wird mittels der Zentrifugalmaschine unter der Drehungsgeschwindigkeit von ca. 4000 pro Minute ausgeführt. Nach der Wegnahme der Flüssigkeit wird der Niederschlag im getrockneten Zustand im Exsikkator erhalten.

b) Die biologische Bestimmungsmethode der geringen Quantität des Insulins.

Zum Zweck der Erhöhung der Empfindlichkeit des Tiers gegen Insulin wurden einige Änderungen in der Krampfmethode der Maus, welche in der britischen ${ }^{11)}$ und internationalen Pharmakopöe verwendet wird, gemacht. Nach der Adrenalektomie werden die Mäuse von dem dd-Stamm, deren Körpergewicht $15 \mathrm{~g} \pm 2 \mathrm{~g}$ ist, 72 Stunden lang im Zimmer von beständiger Temperatur $25^{\circ} \mathrm{C} \pm 2^{\circ} \mathrm{C}$ mit der physiologischen Kochsalzlösung, dem bestimmten Futter und dem Gemüse gezüchtet. Die Enthaltung von Futter findet 5 Stunden lang vor dem Gebrauch statt. Diese Mäuse werden in 3 Gruppen geteilt. Jede Gruppe besteht aus 30 Mäusen. Man injiziert den 2 Gruppen die 2 Dosen von Standardinsulin im Verhältnis von 1 (0.05 Milli-Einheit/10g Körpergewicht) zu 5 (0.25M.E./10g Körpergewicht) oder 1 zu 10. Der anderen Gruppe wird die bestimmte Menge des extrahierten Insulins aus dem Blutplasma, welche dem Blutplasma von $0.02 \mathrm{ccm} \sim 0.05 \mathrm{ccm}$ pro $10 \mathrm{~g}$ Körpergewicht der Maus entspricht, eingespritzt. Die Injektion wird jedenfalls unter die Haut gegeben. Die Menge der zur Injektion gebrauchten Flüssigkeit ist stets $0.25 \mathrm{ccm}$ pro $10 \mathrm{~g}$ Körpergewicht. Nach der Injektion beobachtet man 50 Minuten lang die hypoglykämichen Symptome der Mäuse, die unter $38^{\circ} \mathrm{C}$ im Brutapparat aufgenommen worden sind. Der Krampf, das Koma oder der Kollaps, in welchem die auf den Rücken liegende Maus mehr als 3 Sekunden dieselbe Lage anhält und der Tod werden als positiv beurteilt. Aus dem Prozentsatz der Positivität in jeder Gruppe wird die Einheit des extrahierten Insulins mittels der Finney'sche Rechnungsmethode berechnet.

2) Die Verabreichung von oralen Antidiabetica an Hunde und Ratten.

a) Einige Gruppen der Ratte vom Wistar-Stamm, denen 24 Stunden lang das Futter entzogen wurde, werden getrennt mit $200 \mathrm{mg} / \mathrm{kg}$ von Ca-Mesoxalat, $200 \mathrm{mg} / \mathrm{kg}$ von BZ 55, $200 \mathrm{mg} / \mathrm{kg}$ von D860, $100 \mathrm{mg} / \mathrm{kg}$ von DBI und gebranntem Wasser als Vergleich durch die Metallsonde gefüttert.

Jede Gruppe besteht aus ca. 20 30 Ratten. Nach den bestimmten Stunden werden die Ratten getötet. Das Blut jeder 3 5 Ratten wird in einem Glas gesammelt. Aus dem getrennten Plasma oder Serum wird das Insulin, wie oben erwähnt, extrahiert.

Vol. 35 No. 1 
Das extrahierte Roh-Insulin wird nach der obigen Bestimmungsmethode geprüft.

b) $200 \mathrm{mg} / \mathrm{kg}$ von Ca-Mesoxalat, $200 \mathrm{mg} / \mathrm{kg}$ von BZ55 und $10 \mathrm{mg} / \mathrm{kg}$ von DBI werden getrennt in die Venen der Vorderbeine der Hunde, denen 24 Stunden lang das Futter entzohen wurde, eingespritzt. Nach der Injektion wird das Blut aus der Vene stundenweise gewonnen. Der Insulingehalt im Blut wird, wie oben erwähnt, geprüft.

\section{Ergebnisse}

1) Mesoxalat

Wie die Tabellen I und II zeigen, lässt sich die Zunahme des Insulingehaltes im Blut 2 Stunden nach der Injektion oder der Sondenfütterung bemerken. Die vierfache Zunahme bei Ratten und die dreifache bei Hunden. Aber der Grad der Zunahme ist nach dem Individuum der Hunde ziemlich abweichend.

Tabelle I : Der Blutinsulingehalt der Ratten 2 Stunden nach der Verabfolgung von CaMesoxalat durch die Metallsonde.

\begin{tabular}{c|c}
\hline Aq. dest. & $\begin{array}{c}\text { Ca-Mesoxalat } \\
200 \mathrm{mg} / \mathrm{kg} \mathrm{K.G.}\end{array}$ \\
\hline 6.05 & 20.21 \\
6.35 & 10.08 \\
8.00 & 32.02 \\
1.94 & 31.12 \\
4.24 & 5.04 \\
\hline M.m. 5.31 & 19.69 \\
\hline
\end{tabular}

Die Zahlen zeigen die MilliEinheiten von Insulin pro $1 \mathrm{ccm}$ von Blutplasma.
Tabelle II : Der Blutinsulingehalt der Hunde nach der intravenösen Injektion von $\mathrm{Ca}$ Mesoxalat.

\begin{tabular}{r|c|c|c}
\hline \multirow{2}{*}{ Vor d. Inj. } & \multicolumn{3}{|c}{$\begin{array}{c}\text { Nach d. Inj. von Ca- } \\
\text { Mesoxalat (200mg/kg K.G.) }\end{array}$} \\
\cline { 2 - 4 } & 30 Min. & 60 Min. & 120 Min. \\
\hline A 3.31 & 2.59 & 4.17 & 5.21 \\
B 5.39 & 2.83 & 7.36 & 25.46 \\
C 6.59 & 5.09 & 6.25 & 15.02 \\
\hline M.m. 5.09 & 3.50 & 5.93 & 15.23 \\
\hline Die Zahlen zeigen die Milli-Einheiten von \\
Insulin pro 1 ccm von Blutplasma.
\end{tabular}

2) $\mathrm{BZ} 55$

Wie die Tabellen III und IV zeigen, ist die Zunahme des Insulingehaltes im Blut nach der Verabreichung von BZ55 an Ratten und Hunden nicht bemerkbar. Die Durchschnittszahlen in 3 Stunden und 5 Stunden nach der Verabfolgung von BZ55 an Ratten sind zwar grösser als die Zahl der Gruppe ohne BZ55-Verabfolgung, aber man kann das als die signifikante Differenz nicht erkennen.

3) D860

Wie die Tabelle $\mathrm{V}$ zeigt, ist keine Zunahme des Insulingehaltes im Blut nach der Verabfolgung von D860 an Ratten bemerkbar.

4) DBI (N'-beta-Phenylaethyl-biguanid-hydrochlorid)

Wie die Tabelle VI zeigt, ist keine Zunahme des Insulingehaltes im Blut nach der Verabfolgung von DBI an Ratten bemerkbar. Die Verabfolgung von DBI an 3 Hunde 
Tabelle III : Der Blutinsulingehalt der Ratten nach der Verabfolgung von BZ55 durch die Metallsonde.

\begin{tabular}{r|c|c}
\hline \multirow{2}{*}{ Aq. dest. } & \multicolumn{2}{|c}{$\begin{array}{c}\text { Nach d. Verabfolgung von } \\
\text { BZ55 (200mg/kg K.G.) }\end{array}$} \\
\cline { 2 - 3 } & 3 Std. & 5 Std. \\
\hline 2.00 & 2.42 & 1.64 \\
2.00 & 4.25 & 3.66 \\
6.77 & 5.62 & 6.62 \\
3.02 & 5.43 & 3.67 \\
\hline M.m. 3.4 & 4.4 & 3.8 \\
\hline
\end{tabular}

Die Zahlen zeigen die Milli-Einheiten von Insulin pro $1 \mathrm{ccm}$ von Blutplasma.
Tabelle IV: Der Blutinsulingehalt der Hunde nach der intravenösen Injektion von BZ55.

\begin{tabular}{rr|c|c|c|c}
\hline \multirow{2}{*}{ Vor d. Inj. } & \multicolumn{3}{|c}{$\begin{array}{c}\text { Nach d. Injektion von BZ55 } \\
\text { (200mg/kg K.G.) }\end{array}$} \\
\cline { 2 - 6 } & & 30 Min. & 60 Min. & 120 Min. & 180 Min. \\
\hline A & 2.93 & 2.42 & 3.52 & 1.08 & \\
B & 3.67 & & 2.07 & 1.27 & 2.46 \\
C & 3.72 & & 2.59 & 2.00 & 4.03 \\
\hline & 3.44 & 2.42 & 2.72 & 1.44 & 3.24 \\
\hline
\end{tabular}

Die Zahlen zeigen die Milli-Einheiten von Insulin pro $1 \mathrm{ccm}$ von Blutplasma.

Tabelle V: Der Blutinsulingehalt der Ratten nach der Verabfolgung von D860 durch die Metallsonde.

\begin{tabular}{r|c|c}
\hline \multirow{2}{*}{ Aq. dest. } & \multicolumn{2}{|c}{$\begin{array}{c}\text { Nach d. Verabfolgung von } \\
\text { D860 (200mg/kgK.G.) }\end{array}$} \\
\cline { 2 - 3 } & $3 \mathrm{Std}$. & $5 \mathrm{Std}$. \\
\hline 2.77 & 1.97 & 2.59 \\
2.58 & 4.19 & 1.51 \\
6.23 & 2.44 & 8.12 \\
\hline M.m. 3.86 & 2.86 & 4.07 \\
\hline Die Zahlen zeigen die Milli-Einheiten von \\
Insulin pro 1 ccm von Blutplasma.
\end{tabular}

Tabelle VI: Der Blutinsulingehalt der Ratten nach der Verabfolgung von DBI durch die Metallsonde.

\begin{tabular}{r|c|c}
\hline \multirow{3}{*}{ Aq. dest. } & \multicolumn{2}{|c|}{$\begin{array}{c}\text { Nach d. Verabfolgung von } \\
\text { DBI (100mg/kg K.G.) }\end{array}$} \\
\cline { 2 - 3 } & 3 Std. & 5 Std. \\
\hline 2.27 & 3.06 & 5.68 \\
9.21 & 4.93 & 5.77 \\
3.49 & 2.91 & 4.16 \\
\hline M.m. 4.99 & 3.63 & 5.20 \\
\hline
\end{tabular}

Die Zahlen zeigen die Milli-Einheiten von Insulin pro $1 \mathrm{ccm}$ von Blutplasma.
Tabelle VII : Der Blutinsulingehalt der Hunde nach der intravenösen Injektion von DBI.

\begin{tabular}{|c|c|c|c|}
\hline \multirow{2}{*}{ Vor d. Inj. } & \multicolumn{3}{|c|}{$\begin{array}{l}\text { Nach d. Injektion von } \\
\text { DBI }(10 \mathrm{mg} / \mathrm{kg} \mathrm{K.G.}\end{array}$} \\
\hline & 1 Std. & 3 Std. & 5 Std. \\
\hline A 3.86 & 1.99 & 2.38 & 2.77 \\
\hline B 4.60 & 2.11 & 2.20 & 2.57 \\
\hline C 2.19 & 1.20 & 5.62 & 4.13 \\
\hline M.m. 3.53 & 1.76 & 3.39 & 3.15 \\
\hline
\end{tabular}

Die Zahlen zeigen die Milli-Einheiten von Insulin pro $1 \mathrm{ccm}$ von Blutplasma. 
verursachte die Zunahme des Insulingehaltes im Blut in einem Hunde (Taballe VII). Aber man soll dieses abweichende Resultat nicht als eine allgemein geltende Erscheinung betrachten, da die innere Sakretion von Insulin durch mehrere Fektoren im Körper kontrolliert ist.

\section{Diskussion}

1) Über die Bestimmungsmethode des Insulingehaltes im Blut.

Obgleich von vielen Forschern über mehrere Bestimmungsmethoden in bezug auf den Insulingehalt im Blut berichtet worden ist, so sind es doch nur einige ${ }^{12313) 14}$, welche die Beseitigung der Antagonisten gegen das Insulin aus dem Blutplasma versuchten, die sich an der Regulierung des Blutzuckers antagonistisch gegen das Insulin beteiligen. Ungewöhnliche Anstrengungen in der Forschung über die Erhöhung der Empfindlichkeit des Tieres gegen das Insulin sind zwar sowohl in der Blutzuckermethode ${ }^{15) 16(1) 17)(1) 193}$ als auch in der Zwerchfellmethode ${ }^{\left.2022122322322_{24}\right)}$ gemacht worden, aber auf die Beseitigung der antagonistischen Faktoren gegen das Insulin im Blut wurde nicht geachtet. Es lässt sich jetzt nichts dagegen sagen, dass die Hormon, wie das Adrenalin, das Glukagon, die Glukokortikoide und das Wachstumshormon, eine antagonistische Wirkung gegen das Insulin bei der Regulierung des Blutzuckers haben, nach welcher der Blutzucker und der Zuckerstoffwechsel im Körper nach der Veränderung des äusseren Milieus richtig reguliert werden. Wir glauben, dass die Beseitigung der antagonistischen Faktoren gegen das Insulin aus dem Blut durch das Verfahren der Extraktion die unentbehrliche Bedingung bei der Bestimmung des Insulingehaltes im Blut ist ; denn die hypoglykämischen Krämpfe der 72-Stunden-adrenalektomierten Mäuse werden durch das dem Blutplasma von $0.025 \mathrm{ccm}$ pro $10 \mathrm{~g}$ Körpergewicht entsprechende extrahierte Insulin verursacht, während das Blutplasma selbst von $0.5 \mathrm{ccm}$ pro $10 \mathrm{~g}$ Körpergewicht bei diesen Mäusen keinen Krampf und kein Koma verursachen kann.

Diese Tatsache erläutert den Grund des fast zehnfach höheren Bestimmungswertes des Insulingehaltes im Blut mittels unserer Methode im Vergleich mit den anderen Methoden, die das Verfahren der Extraktion nicht anwenden.

2) Über den Wirkungsmechanismus von Mesoxalat.

1952 entdeckten Kobayashi ${ }^{()}$et al., Ōhashi ${ }^{\text {(7s) }}$ et al. die blutzuckersenkende Wirkung von Mesoxalat,und sie glaubten, dass diese Stoffe mittels der Reizung der B-Zellen der Langerhans'schen Inseln die peripherische Benutzung des Zuckers steigern. Ōhashi ${ }^{25}$ et al. stützten diese Meinung auf den histologischen Befund der Langerhans'schen Inseln der verschiedenartigen Tiere, denen auf eine lange Zeit Mesoxalat verabfolgt wurde. Takeuchi $^{262 \text { 2 })}$ berichtete, dass die langfristige Verabfolgung von Mesoxalat die Zunahme des Insulingehaltes in dem Pankreas der Ratten und der Hunde verursacht. Diese Berichte stimmen in bezug auf den Wirkungsmechanismus mit unserem Resultat überein. Bei einmaliger Verabfolgung des Mesoxalates von $200 \mathrm{mg} / \mathrm{kg}$ Körpergewicht lassen sich die vierfache Zunahme des Blutinsulingehaltes bei Ratten und die dreifache bei Hunden verursachen. 
Nach diesen Tatsachen besteht kein Zweifel, dass Mesoxalat mittels der Reizung der B-Zellen der Langerhans'schen Inseln die Sekretion des Insulins in den Blutstrom steigert. Und es ist nichts anderes als die Ergänzung des obigen Wirkungsmechanismus, dass Mesoxalat bei klinischen Verwendungen die gleichen Einflüsse auf die peripherische Benutzung des Zuckers wie das Insulin hat.

3) Über den Wirkungsmechanismus von Sulfonylurea.

Obgleich Sulfonylurea zur Behandlung der Zuckerkrankheiten verwendet wird, da es blutzuckersenkende Wirkung hat, so ist doch der Wirkungsmechanismus dieses Stoffs noch unklar.

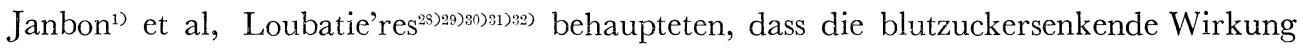
von Sultonylurea auf die Steigerung der Insulinsekretion aus den B-Zellen der Langerhans'schen Inseln zurückzuführen ist, da die Wirkung dieser Stoffe auf den Blutzuckerwert mittels der Pankreatektomie verloren geht. Volk ${ }^{33)}$ et al. stützten diese Meinung auf den histologischen Befund der B-Zellen, welche die deutliche Degranulierung am vierten Tag der täglichen Verabfolgung von D860 und am achten Tag nahezu vollständige Entgranulierung aus dem Zytoplasma zeigten.

Mittels der Färbungsmethode nach Gomori und der Elektromikroskopie stützten auch Yoshida $^{3+)}$ et al. diese Meinung auf den histologischen Befund der B-Zellen eines Kaninchens, welche zwischen anderthalb und 3 Stunden nach der einmaligen Sondenfütterung von BZ55 (250mg/kg) deutliche Degranulierung zeigten. Und auch Colwell ${ }^{35)}$ et al. sind derselben Meinung über den Wirkungsort von Sulfonylurea; denn die stärkste Senkung des Blutzuckers tritt im Falle der Einspritzung von D860 in die pankreatiko-duodenale Arterie ein im Vergleich mit den anderen Fälle, in welchen die Injektionen in die hepatische Arterie, in die Pfortader und in die Femoralvene gemacht werden.

Da die Zunahme des Insulingehaltes im Blut nach der Verabfolgung von Sulfonylurea in den obenangeführten Berichten nicht festgestellt wurde, kann man nicht sagen, dass diejenigen Behauptungen, welche die blutzuckersenkende Wirkung von Sulfonylurea dem Steigen der Sekretion des Insulins aus den B-Zellen zuschreiben, wohlbegründet sind; denn es ist unrichtig, das Steigen der Sekretion des Insulins aus der Degranulierung der B-Zellen zu schliessen, wie Wrenshall ${ }^{36)}$ et al. und Haist ${ }^{37}$ berichteten, wenn das Steigen und Fallen von B-Zell-Granula auch den Wechsel des Insulingehaltes in den B-Zellen bedeutet: d.h. die Degranulierung der B-Zellen lässt sich nicht nur im Falle des Steigens der Ausströmung des Insulins aus den B-Zellen in den Blutstrom, sondern auch im Falle der Verminderung der Produktion des Insulins in den B-Zellen verursachen.

Wir bestätigten mittels der neuen Bestimmungsmethode des Insulingehaltes im Blut, dass Sulfonylurea die Zunahme der Sekretion des Insulins aus dem Pankreas nicht verursachen kann. Diese Folgerung ist im Widerspruch mit den Resultaten von Holt ${ }^{38)}$ et al und Kracht ${ }^{393}$ et al, welche im Experiment, das die Glykogensynthese im Rattenzwerchfell als das Zeichen der Insulinaktivität benutzt, die sechsfache oder die siebenfache Zunahme der insulinartigen 
Aktivität des Blutplasmas nach der Verabfolgung von BZ55 oder D860 andeuten.

Aber es dünkt uns unglaubwürdig; denn die Spezifität gegen das Insulin und die vertrauenswürdige Schranke der Methode sind nicht erwähnt, und die Beseitigung der Antagonisten gegen das Insulin im Blutplasma wurde nicht vorgenommen.

Mehrere Berichte, welche die Uneinigkeit der Wirkungen von Insulin und Sulfonylurea im peripherischen Stoffwechsel andeuten, mögen unsere Folgerung unterstützen.

Elrick ${ }^{40)}$ et al berichteten, dass keine Zunahme von A-V/A (die arteriovenöse GlukoseDifferenz/die arterielle Glukose-Konzentration) durch die Verabfolgung von D860 verursacht wird, dagegen dass eine deutliche Zunahme von A-V/A nach der Verabfolgung von Insulin bemerkbar ist. Auch Recant ${ }^{41)}$ et al prüften die Einwirkung von Sulfonylurea auf A-V/A und bekamen kein Resultat, welches das Steigen der Benutzung des Zuckers im peripherischen Gewebe andeutet. Sie halten dafür, dass die Hemmung der Ausströmung des Traubenzuckers aus der Leber in den Blutstrom den Wirkungsmechanismus der Senkung des Blutzuckers erkläre. Sie sind der Meinung, dass der Wirkungsort von Sulfonylurea hauptsächlich in der Leber sein müsse, in welcher dieser Stoff zur Verstärkung der Wirkung des Insulins mit demselben zusammenwirken müsse; denn es zeigt nichts anderes als die Steigerung der Benutzung des Kohlenhydrates in der Leber, dass sich nach der. Verabfolgung von Sulfonylurea die Zunahme der Benutzung der Aminosäure in dem Leberschnitt und die Vermehrung der Brenztraubensäure in der hepatischen Vene bemerken lassen.

Auch Renold ${ }^{42)}$ et al sind der Meinung, dass der Mechanismus der Senkung des Blutzuckers nicht in der Steigerung der Benutzung des Zuckers im peripherischen Gewebe, sondern in der Einwirkung von Sulfonylurea auf die Leber, in welcher dieser Stoff auf die Glykogensynthese oder auf die Ausströmung des Zuckers in den Blutstrom einen Einfluss habe, bestehen müsse; denn sie bemerkten die folgende Tatsache, dass in der Zwerchfellmethode die insulinartige Aktivität des Blutplasmas von den D860-injizierten Menschen im Vergleich mit der des vor der Injektion gewonnenen Blutplasmas keine Erhöhung zeigt.

Hierauf ist die hepatotrope Theorie als der Wirkungsort von Sulfonylurea akzentuiert. Mohnike $^{43}$ ) et al. schlossen von der Tatsache, dass die Verminderung von Kalium, Phosphor und Amino-Stickstoff im Blutserum nach der Verabfolgung von Sulfonylurea nur gering und mit dem Stadium der Senkung des Blutzuckers nicht so sehr im Einklang sind, trotz der deutlichen Verminderung dieser Stoffe und der guten Übereinstimmung mit dem Stadium im Falle der Verabfolgung von Insulin, darauf, dass Sulfonylurea gegen Insulin den peripherischen Stoffwechsel kaum oder nicht steigern.

Als andere Hypothesen lassen sich die folgenden angeben; 1) die Theorie der A-ZellenStörung oder die Theorie des Hemmnisses der Glukagon-Aktivität (Bertram ${ }^{1)}$ et al.,

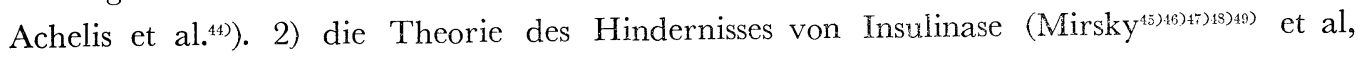
Williams $^{5051)}$ et al.). 3) die Theorie des Hindernisses der Glykogenolyse in der Leber (Vaughan $^{52)}$, Moorhouse ${ }^{53}$ et al.). Die erste Theorie wurde von Volk ${ }^{33)}$ et al. verneint, 
In bezug auf die zweite Theorie führten die Experimente, in welchen Volk ${ }^{33)}$ et al. und Wick $^{54}$ et al. die Zerlegung von ${ }^{131}$-Insulin im Körper prüften, zu den versagenden Resultaten.

Vaughan, der die dritte Theorie aufstellte, ist der Meinung, dass Sulfonylurea die Hemmwirkung auf die Phosphokinase ausübe; denn D860 hemmt die durch das Adrenalin oder Glukagon verursachte Ausströmung der Glukose aus der Leber in den Blutstrom.

Obwohl das Wesen des Wirkungsmechanismus von Sulfonylurea, wie oben erwähnt, noch nicht erklärt ist, so liefert unser Experiment doch den Beweis, dass Sulfonylurea die Zunahme der Sekretion des Insulins aus dem Pankeras nicht verursachen kann.

\section{Zusammenfassung}

Der Verfasser untersuchte die Wirkung von oralen Antidiabetica auf den Blutinsulingehalt der Hunde und der Ratten mittels der neuen Methode über die Bestimmung des Blutinsulingehaltes und bekam die folgenden Resultate.

1) Mesoxalat steigert die Ausströmung des Insulins aus dem Pankreas in den Blutstrom.

2) Sulfonylurea (BZ55 oder D860) verursacht keine Steigerung der Ausströmung des Insulins aus dem Pankreas in den Blutstrom.

3) DBI verursacht keine Steigerung der Ausströmung des Insulins aus dem Pankreas.

4) Die Diskussion über den Wirkungsmechanismus von Mesoxalat und Sulfonylurea wurde ausgeführt.

5) Eine Diskussion wurde über die Bestimmungsmethode des Insulingehaltes im Blut ausgeführt.

\section{Summary}

The author studied the effects of oral antidiabetica on the blood insulin content of dogs and rats by the new method for determination of the blood insulin and obtained the following results.

1) Mesoxalate elevate the release of the insulin from the pancreas in the blood stream.

2) Sulfonylurea (BZ55 or D860) causes no elevation of the release of the insulin from the pancreas in the blood stream.

3) DBI causes no elevation of the release of the insulin from the pancreas.

4) The mechanisms of the action of mesoxalate and sulfonylurea were discussed.

5) The methods for the determination of the insulin content in the blood were discussed.

Anerkennung; Ich möchte Herrn Dr. T. Minesita vielen Dank für seine freundliche Leitung in dieser Arbeit und Herrn E. Itoga Dank für seine mühevolle technische Hilfe aussprechen.

\section{Literatur}

1) Janbon, M., J. Chaptal, A. Vedel \& J. Schaap, Montpellier med, 1942, 21-22; 441

2) Franke, H., J. Fuchs, Deutsch. med. Wochenschr. 1955, $80: 1450$ 
3) Achelis, J.D., E. Haack, K. Hardebeck, Deutsch, med. Wochenschr., 1955, 80 : 1452

4) Bertram, F., E. Bendfeldt, H. Otto, Deutsch, med. Wochenschr., 1955, $80: 1455$

5) Erhart, G., Naturwissenschaft, 1956, 43 ; 93

6) Kobayashi, Y., S. Ohashi, S. Takeuchi, Folia Pharmacol. Japon., 1952, 48 : 348

7) Ohashi, S., S. Takeuchi, Y. Kobayashi, Folia Pharmacol. Japon., 1952, $48: 358$

8) Ohashi, S., S. Takeuchi, Y. Kobayashi, Folia Pharmacol. Japon., 1953, 49 : 143

9) Minesita, T., E. Itoga, G. Nagata, A. Tanaka, Proceedings of the Japan Academy, 1958, V. 34, N. $3: 177$

10) Best, C.H., C.M. Jephcott, D.A. Scott, Am. J. Physiol., 1932, 100 : 285

11) British Pharmacopoeia, $1958: 936$

12) Hoshi, T., Tōhoku Igaku., 1926, $9: 73$

13) Baird, G.W., J. Bornstein, Lancet, I. 1957 : 1111

14) Biegelman, P.M., H.N. Antoniades, F.C. Goetz, A.E. Renold, J.L. Oncley, G.W. Thorn, J. Clin. Exp. Metabol., 1956, $5: 44$.

15) Allen, A., J. Feldman, E. Gellhorn, Am. J. Physiol., 1941, 133 : 193

16) Gellhorn, E., J. Feldman, A. Allen, Endocrinology, 1941, $29: 137$

17) Anderson, E., E. Lindner, V. Sutton, Am. J. Physiol., 1947, $149: 350$

18) Bornstein, J., Austr. J. Exp. Biol \& Med. Sci., 1950, $28: 87$

19) Kosaka, K., Folia Endocrinol. Japon., 1957, V. 33, N. $7: 571$

20) Perlmutter, M., S. Weisenfeld, M. Mufson, Endocrinology, 1952, $50: 442$

21) Groen, J., C.E. Kamminga, A.F. Willebrands, J.R. Blickman, J. Clin. Investig. 1952, $31: 97$

22) Vallance- Owen, J., B. Hurlock, Lancet I. $1954: 68$

23) Takeuchi, S., Ōhashi, Y. Kobayashi, Folia Pharmacol. Japon., 1957, 53 : 740

24) Takeuchi, S., S. Ōhashi, Y. Kobayashi, Folia Pharmacol. Japon., 1957, 53 : 747

25) Ōhashi, S., S. Takeuchi, Y. Kobayashi, K. Ikeda, Folia Pharmacol. Japon., 1953, 49 : 151

26) Takeuchi, S., Folia Pharmacol. Japon., 1955, V. 51, N. $6: 676$

27) Takeuchi, S., Endocrinol. Japon., 1958, V. 5, N. $3: 177$

28) Loubatieres, A., Compt. rend. Soc. biol., 1944, $138: 766$

29) Loubatieres, A, These Doet. Sci. naturelles Montpellier, 1946, No. 86

30) Loubatieres, A., Arch. intern. Physiol., 1946, 54 : 174

31) Loubatieres, A., Annals N.Y. Academy Scien., 1957, $71: 4$

32) Loubatieres, A., Annals N.Y. Academy Scien., 1957, $71: 192$

33) Volk, B.W., M.G. Goldner, S. Weisenfeld, S.S. Lazarus, Annals N.Y. Academy Scien., 1957, $71: 141$

34) Yoshida, H., S. Yokoo, O. Aochi, T. Uehira, Folia Endocrinol. Japon., 1958, V. 34, $1: 1$

35) Colwell, A.R., Jr, J.A. Colwell, A.R. Colwell, Sr., Annals N.Y. Academy Scien., 1957, 71 : 125

36) Wrenshall, G.H., S.A. Hartroft, C.H. Best, Diabetes, 1954, $3: 444$

37) Haist, R.E., Physiol. Revs., 1944, 24 : 409

38) v. Holt, C., L. v. Holt, J. Kracht, B. Kröner, Science, 1957, 125 : 735

39) Kracht, J., B. Kröner, L. v. Holt, C.v. Holt, Naturwissenschaft, 1957, 44 : 16

40) Elrick, H., R. Purnell, Annals N.Y. Academy Scien., 1957, $71: 38$

41) Recant, L., G.L. Fischer, Annals N.Y. Academy Scien., 1957, $71: 62$

42) Renold, A.E., D.B. Martin, B.B. Boshell, G.W. Thorn, Annals N.Y. Academy Scien., 1957, 71 ; 71

43) Mohñike, G., A. Czyzyk, H. Bibergeil, Arzneimittelforschung, 1958, Juli : 475 
44) Achelis, J.D., K. Hardebeck, Deutsch, med. Wochenschr., 1955, 80 : 1452

45) Mirsky, I.A., Metabolism, 1956, $5: 138$

46) Mirsky, I.A., D. Diengott, G. Perisutti, Endocrinology, 1956, $59: 715$

47) Mirsky, I.A., G. Perisutti, D. Diengott, Metabolism, 1956, 5 : 156

48) Mirsky, I.A., D. Diengott, H. Dolger, Science, 1956, 123 : 583

49) Mirsky, I.A., D. Diengott, Proc. Soc. Exp. Biol. a. Med., 1956, 93 : 109

50) Williams, R.H., Metabolism, 1956, $5: 128$

51) Williams, R.H., M.K. Berg, Proc. Soc. Exp. Biol. a. Med., 1956, 92 : 20

52) Vaughan, M., Science 1956, $123: 885$

53) Moorhouse, J.A., R.M. Kark, Clin. Research Proc., 1956, 4 : 124

54) Wick, A.W., M. Karasek, B. Britton, Annals N.Y. Academy Scien., 1957, 71 : 35 